\title{
Article \\ Evaluation of an Augmented Cognitive Behavioural Group Therapy for Perinatal Generalized Anxiety Disorder (GAD) during the COVID-19 Pandemic
}

\author{
Sheryl M. Green ${ }^{1,2, *}$, Briar Inness ${ }^{2,3}$, Melissa Furtado ${ }^{2,3}$, Randi E. McCabe ${ }^{1,4}$ and Benicio N. Frey ${ }^{1,2}$ \\ 1 Department of Psychiatry and Behavioural Neurosciences, McMaster University, \\ Hamilton, ON L8N 3K7, Canada; rmccabe@stjoes.ca (R.E.M.); bfrey@stjoes.ca (B.N.F.) \\ 2 Women's Health Concerns Clinic, St. Joseph's Healthcare, Hamilton, ON L8N 3K7, Canada; \\ innessbe@mcmaster.ca (B.I.); furtadom@mcmaster.ca (M.F.) \\ 3 Department of Psychology, Neuroscience and Behaviour, McMaster University, \\ Hamilton, ON L8N 3K7, Canada \\ 4 Anxiety Treatment and Research Clinic, St. Joseph's Healthcare, Hamilton, ON L8N 3K7, Canada \\ * Correspondence: sgreen@stjoes.ca; Tel.: +1-905-522-1155 (ext. 33672)
}

\section{check for}

updates

Citation: Green, S.M.; Inness, B.;

Furtado, M.; McCabe, R.E.; Frey, B.N.

Evaluation of an Augmented

Cognitive Behavioural Group Therapy for Perinatal Generalized

Anxiety Disorder (GAD) during the COVID-19 Pandemic. J. Clin. Med.

2022, 11, 209. https://doi.org/

10.3390/jcm11010209

Academic Editors: Eva Meler and Anna Goncé

Received: 14 November 2021

Accepted: 29 December 2021

Published: 31 December 2021

Publisher's Note: MDPI stays neutral with regard to jurisdictional claims in published maps and institutional affiliations.

Copyright: (c) 2021 by the authors Licensee MDPI, Basel, Switzerland. This article is an open access article distributed under the terms and conditions of the Creative Commons Attribution (CC BY) license (https:/ / creativecommons.org/licenses/by/ $4.0 /)$.

\begin{abstract}
The perinatal period is considered a window of vulnerability given the increased risk of psychiatric difficulties during this time, such as mood and anxiety disorders (ADs). Pre-pandemic rates of ADs in perinatal women were one in five but have since increased with the onset of the COVID-19 pandemic (COVID). In addition, recent research suggests that the focus of worry has shifted during the pandemic, with perinatal women reporting significantly more COVID-specific worries. The objective of this study was to augment our current evidence-based Cognitive Behavioural Group Therapy (CBGT) for perinatal anxiety protocol by targeting intolerance of uncertainty and tailoring existing strategies to address COVID-related worry and impact. Pregnant $(n=19)$ and postpartum $(n=49)$ women were recruited from regular clinic patient flow from a university-affiliated teaching hospital between September 2020 and March 2021. Improvements in generalized anxiety symptoms, worry, intolerance of uncertainty, and mood were observed at post-treatment, maintained at 3-months, and the intervention received high ratings of treatment satisfaction. This is the first study to examine an augmented CBGT for perinatal women with GAD during the pandemic and supports the inclusion of strategies that target intolerance of uncertainty as well as specific pandemic and perinatal worry content for effective outcomes.
\end{abstract}

Keywords: perinatal GAD; COVID-19 pandemic; Cognitive Behavioural Group Therapy; augmented treatment

\section{Introduction}

Pregnancy and the postpartum, often referred to as the perinatal period, are associated with increased risk of experiencing psychiatric difficulties such as mood and anxiety disorders [1,2]. Anxiety Disorders (ADs), in particular, affect up to one in five pregnant and postpartum women [3]. Since the onset of the COVID-19 pandemic (COVID), perinatal women appear to be at an even greater risk of experiencing significant anxiety, with $43-60 \%$ of perinatal women endorsing moderate to severe levels of generalized anxiety symptoms [4,5]. Given that perinatal ADs are associated with numerous adverse outcomes for both mothers and their infants [6-10], this increase in anxiety symptom severity is concerning.

Generalized Anxiety Disorder (GAD), characterized by excessive and difficult to control worry [11], is the most common AD in pregnancy and the postpartum period [3,12] While people with GAD tend to endorse similar worry domains across the lifespan (e.g., worry about health or self and others, finances, work/school [13]), the focus of worry often shifts to reflect the current context and circumstance of one's life [14-16]. The perinatal 
period is no exception, as pregnant and postpartum women with GAD tend to endorse worries that are predominantly maternally focused (e.g., parenting abilities, coping as a mother, the well-being of the infant [17], reflecting the current developmental context of their lives. Further, pandemic-specific anxiety has been documented in the general population (e.g., fear of infection [18]) and in perinatal samples (e.g., exposure risks for mother and baby; reduced social support; uncertainty of perinatal care $[4,19])$, suggesting that worry is also circumstance specific.

Given that the prevalence of perinatal anxiety has increased substantially since the onset of COVID, and the focus of worry often reflects the current context (e.g., perinatal) and circumstance (e.g., COVID) of life, our team qualitatively examined the worry content of 84 treatment seeking pregnant and postpartum women during the pandemic (between April 2020 to October 2020), the majority (94\%) of which, had a diagnosis of GAD [20]. We found that $33.5 \%$ of participant's principal worries were specific to COVID, and $40 \%$ of those COVID worries were specific to the perinatal context. Further, our results revealed that a significant number of COVID worries were related to reduced social support and uncertainty about the future. This is troublesome as both reduced social support and intolerance of uncertainty are associated with worsening of postpartum depression and anxiety symptoms [21-23].

Intolerance of uncertainty, defined as a dispositional characteristic that results from a set of negative beliefs about uncertainty and its implications and involves the tendency to react negatively on an emotional, cognitive, and behavioral level to uncertain situations and events [24], is associated with heightened distress and worry [25-27]. Individuals who are intolerant of uncertainty see ambiguity as stressful and anxiety provoking and believe that uncertain situations should be avoided. Further, they have the tendency to overestimate the possibility of unpredictable or negative events and make threatening interpretations of ambiguous information. Unsurprisingly, uncertainty has increased since the onset of the pandemic, as COVID represents an unprecedented challenge for everyone [28], but may be particularly challenging for those who are already intolerant of uncertainty. For instance, a recent study by Sbrilli and colleagues [29], found that elevated levels of intolerance of uncertainty in pregnant and postpartum women during the pandemic were associated with decreased self-reported mindfulness and increased psychological symptoms, such as depression and anxiety. Importantly, interventions that specifically include strategies targeting intolerance of uncertainty (e.g., behavioural experiments, mindfulness) have demonstrated reductions in worry and anxiety in non-perinatal populations [30-32]. As such, the need to target intolerance of uncertainty in perinatal treatment protocols is needed now more than ever and indeed the call to include them when adapting current protocols during the pandemic, has been made in recent studies [28].

Knowing that perinatal women with GAD are uniquely impacted by the COVID-19 pandemic provides justification for augmenting current treatment strategies to meet their unique mental health needs during this time. To date, no evidence-based psychological treatments have been developed for perinatal women with GAD that specifically target identified COVID-related worry content and its related impact despite the tremendous negative burden of COVID on pregnant and postpartum women [20]. As such, the objective of this study was to augment our current evidence-based Cognitive Behavioural Group Therapy (CBGT) for perinatal anxiety protocol $[33,34]$, by including additional sessions that target intolerance of uncertainty, as well as tailoring existing cognitive and behavioural strategies to address COVID-related worry and impact identified by our qualitative research [20] and evaluate its effectiveness. We hypothesized that this augmented protocol would (1) significantly improve anxiety and worry, both in general and specifically related to COVID-19 specific worries, (2) reduce intolerance of uncertainty, (3) improve mood and (4) result in high treatment satisfaction by perinatal women with GAD. 


\section{Materials and Methods}

\subsection{Participants and Procedures}

Participants were treatment-seeking patients of the Women's Health Concerns Clinic (WHCC), St. Joseph's Healthcare Hamilton, a university-affiliated teaching hospital and publicly funded outpatient clinic that specializes in the assessment and treatment of women's mental health across the reproductive lifespan [35]. All procedures were approved by the Hamilton Integrated Research Ethics Board. Pregnant $(n=19)$ and postpartum $(n=49)$ women recruited from regular clinic patient flow, participated in this study. Pre-treatment assessments took place between September 2020 and March 2021. Eligibility criteria were as follows: (1) 18-45 years old; (2) pregnant or within the first 12 months postpartum; (3) a principal diagnosis of GAD confirmed through use of the Mini International Neuropsychiatric Interview for DSM-5 [36] and (4) fluent in English. Exclusion criteria were as follows: (1) active suicidal ideation and (2) current psychosis or substance use disorder. Assessments were completed by PhD-level clinical psychology graduate students with extensive training in semi-structured diagnostic assessments, and diagnoses were confirmed by a supervising licensed clinical psychologist. Participants also completed a demographic form (see Table 1 for participant sample details) along with a brief battery of questionnaires to further characterize the symptomatology of our sample. Self-report measures were re-administered within one to two weeks post-treatment, along with a treatment satisfaction questionnaire. In order to capture participants' subjective interpretations of their improvements in COVID-related worry across treatment, participants were also asked to identify whether they were still experiencing excessive COVID worry and whether treatment effectively addressed their COVID worries at the post-treatment assessment. Finally, baseline questionnaires were readministered at three-months post-treatment to assess sustained symptom change. Each treatment group was led by a licensed clinical psychologist or senior PhD student in clinical psychology with extensive training in this protocol and co-led with a graduate-level clinical psychology trainee. Treatment groups were composed of 8 sessions, two hours in duration, occurring weekly. Importantly, at the time of data collection from pre-treatment to post-treatment (September 2020 to April 2021) vaccination in Canada had just started to become available as of January 2021. However, prioritization was to healthcare providers and front-line workers and was not readily available to others until later into the spring 2021. Further, the province of Ontario, Canada, where data collection was being conducted, went into lockdown in March 2021 to June 2021 due to the high numbers of COVID-19. As such, although vaccination rates are not known, participants in the study were likely unvaccinated (as availability was minimal to non-existent at the time of pre to post testing) and measures to control the virus (e.g., significant restrictions, lockdown) were in place, throughout the entire study.

\section{Intervention-Augmented CBGT}

The original CBGT treatment, based on our published manual [33] tested in a randomized clinical trial [34], was designed to target a range of anxiety symptoms as well as comorbid depressive symptoms. The traditional CBGT involved 6-weekly 2-h sessions in a small-group format (up to six participants per group; range $=4-6$ ). Session content is tailored to address the unique challenges experienced by women with perinatal anxiety and depression (see [34] for details) with weekly assigned homework exercises designed to reinforce learning. The augmented CBGT protocol (Table 2) contained 2 additional sessions (for a total of 8). These additional sessions provided psychoeducation on COVID-19 (both general and specific perinatal facts), as well as cognitive and behavioural strategies tailored to focus on COVID-specific content. The cognitive and behavioural strategies in the traditional protocol were augmented to include critical COVID-related anxiety, worry, and impact content that was identified by pregnant and postpartum women with GAD in our previous study [20]. The two additional sessions (sessions 5 and 6 in the augmented CBGT) emphasized the role of intolerance of uncertainty in ADs. This content was informed by current CBT for intolerance of uncertainty protocols $[37,38]$, but again, tailored to meet the 
unique needs of perinatal women and included behavioural experiments and mindfulness to increase one's tolerance to uncertainty.

Table 1. Participant Demographics and Characteristics.

\begin{tabular}{|c|c|c|c|}
\hline & Pregnant $(n=19)$ & Postpartum $(n=49)$ & Difference \\
\hline * Age, M(SD) & $30.78(4.88)$ & $30.88(4.33)$ & $\mathrm{t}(65)=-0.08, p=0.94, \mathrm{~d}=0.02$ \\
\hline \multirow[t]{2}{*}{ * Number of children, M(SD) } & $0.56(0.86)$ & $1.35(0.56)$ & $\mathrm{t}(65)=-4.41, p<0.01, \mathrm{~d}=1.09$ \\
\hline & $n(\%)$ & $n(\%)$ & \\
\hline \multicolumn{4}{|l|}{ * Ethnicity } \\
\hline Caucasian & $16(88.9)$ & $43(87.8)$ & \multirow{6}{*}{$\chi^{2}(1)=2.56, p=0.77, \varphi=0.20$} \\
\hline African American & $0(0)$ & $1(2)$ & \\
\hline Asian/Pacific Islander & $0(0)$ & $1(2)$ & \\
\hline Hispanic & $0(0)$ & $1(2)$ & \\
\hline Indigenous & $0(0)$ & $1(2)$ & \\
\hline Other & $2(11.1)$ & $2(4.1)$ & \\
\hline \multicolumn{4}{|l|}{ * Marital status } \\
\hline Single & $1(5.6)$ & $2(4.1)$ & \multirow{2}{*}{$\chi^{2}(1)=0.07, p=0.80, \varphi=0.03$} \\
\hline Married/Common-Law & $17(94.4)$ & $47(95.9)$ & \\
\hline \multicolumn{4}{|l|}{ * Highest education } \\
\hline Some or completed high school & $1(5.6)$ & $3(6.1)$ & \multirow{5}{*}{$\chi^{2}(1)=1.61, p=0.95, \varphi=0.16$} \\
\hline Certificate/Professional Diploma & $10(55.5)$ & $23(46.9)$ & \\
\hline Bachelor's Degree & $4(22.2)$ & $15(30.6)$ & \\
\hline Masters Degree & $3(16.7)$ & $7(14.3)$ & \\
\hline Professional Degree (e.g., MD, JD) & $0(0)$ & $1(2)$ & \\
\hline Comorbid anxiety disorders & $7(36.8)$ & $14(28.6)$ & $\chi^{2}(1)=0.44, p=0.51, \varphi=-0.08$ \\
\hline Comorbid mood disorders & $10(52.6)$ & $32(65.3)$ & $\chi^{2}(1)=0.93, p=0.34, \varphi=0.12$ \\
\hline Current psychotropic medication use ${ }^{* *}$ & $5(26.3)$ & $24(50)$ & $\chi^{2}(1)=3.53, p=0.17, \varphi=0.23$ \\
\hline
\end{tabular}

* 1 pregnant participant did not complete all demographic measures. ${ }^{* *} 1$ postpartum participant did not complete medication history questionnaire.

\subsection{Measures}

\subsubsection{GAD-7 (GAD-7)}

The Generalized Anxiety Disorder 7-Item Scale (GAD-7) [39] is a 7-item self-report questionnaire assessing severity of generalized anxiety symptoms experienced over the previous two-weeks [39]. Items on the GAD-7 are measured on a 4-point Likert scale ranging from 0 (not at all) to 3 (nearly every day). The GAD-7 has demonstrated good sensitivity $(89 \%)$ and specificity $(82 \%)$ in detecting a clinical diagnosis of GAD, when a cut-off score of 10 or higher is utilized [39]. The internal consistency of the GAD-7 in the current sample was $\alpha=0.86$.

\subsubsection{Penn State Worry Questionnaire (PSWQ)}

The PSWQ [40] is a 16-item self-report measure that assesses worry and symptoms characteristic of GAD. Respondents are asked to rate each item on a 5-point scale ranging from 'not typical at all' (1) to 'very typical' (5), with total scores ranging from 16 to 80 and scores at or above 65 representing a clinically significant level of worry [41]. The PSWQ has demonstrated excellent internal reliability and validity across various populations [42], and has been utilized in both non-perinatal and perinatal samples $[43,44]$. The internal consistency of the PSWQ in the current sample was $\alpha=0.78$.

\subsubsection{Intolerance of Uncertainty Scale (IUS)}

The IUS [45] is a 27-item self-administered questionnaire assessing one's beliefs and reactions to uncertain events and ambiguity $[45,46]$. Items are scored on a 5-point Likert scale, ranging from 1 (not at all characteristic of me) to 5 (entirely characteristic of me), with total possible scores of 27 to 135 . The IUS has demonstrated excellent internal consistency 
( $\alpha=0.91-0.95)$ and good test-retest reliability $(\mathrm{r}=0.78)$ in general (non-perinatal) populations. The IUS was recently validated for use as a screening tool for perinatal anxiety disorder risk, in which an optimal clinical cut-off score of 64 or greater was established [47]. The internal consistency of the IUS in this sample was $\alpha=0.92$.

Table 2. Augmented 8-week CBGT protocol session by session content.

\begin{tabular}{|c|c|}
\hline Session & CBGT Augmented Protocol Content \\
\hline 1 & $\begin{array}{l}\text { Introduction and Psychoeducation: Information about anxiety, perinatal anxiety, and impact of COVID-19; } \\
\text { introduction to the cognitive-behavioral model, the role of thoughts in maintaining distress, common } \\
\text { perinatal and COVID-19 related worries and impact, and symptom monitoring. }\end{array}$ \\
\hline 2 & $\begin{array}{l}\text { Identifying and Challenging Unhelpful Thinking: Identifying unhelpful thinking and thinking errors; } \\
\text { introduction to three strategies for cognitive restructuring (i.e., Best Friend Technique, Evidence Technique, } \\
\text { Possibility Pie) to generate more balanced thinking. COVID-19 examples utilized with each technique. }\end{array}$ \\
\hline 3 & $\begin{array}{l}\text { Helpful vs. Unhelpful Worry: Differentiating between productive and unproductive worry; introduction to } \\
\text { a systematic approach to problem-solving for productive worry. COVID-19 examples utilized. }\end{array}$ \\
\hline 4 & $\begin{array}{l}\text { Targeting Problematic Behavior: Psychoeducation on the role that behavior can play in maintaining } \\
\text { distress; identifying problematic behavior (e.g., excessive reassurance seeking, excessive checking, } \\
\text { avoidance with COVID-19 content woven in); introduction to exposure-based behavioral experiments. Use } \\
\text { of COVID-19 related examples to demonstrate strategy. }\end{array}$ \\
\hline 5 (new session) & $\begin{array}{l}\text { Introduction to Myths and Facts of COVID-19 and Intolerance of Uncertainty: Both general and perinatal } \\
\text { themed myths and facts offered along with resources for further facts. Introduction to intolerance of } \\
\text { uncertainty and behavioural experiments to increase tolerance to uncertainty }\end{array}$ \\
\hline 6 (new session) & $\begin{array}{l}\text { In-session Behavioural Experiments and Introduction to Mindfulness: Conducting behavioural } \\
\text { experiments in session and an introduction to mindfulness within session practice as a means to target } \\
\text { intolerance of uncertainty. }\end{array}$ \\
\hline 7 & $\begin{array}{l}\text { Managing Depression: Psychoeducation on depressive symptoms in the perinatal period, risk factors and } \\
\text { prevalence, as well as the impact of COVID-19 on increased risk of depression such as withdrawing from } \\
\text { activity and isolation; impact that hormones and other biological and psychosocial changes have on mood; } \\
\text { introduction to behavioral activation and activity scheduling within the government restrictions and safety } \\
\text { guidelines of the COVID-19 pandemic. Introduction to paced respiration. }\end{array}$ \\
\hline 8 & $\begin{array}{l}\text { Assertive Communication: Psychoeducation on assertive and other forms of communication (i.e., passive, } \\
\text { aggressive, passive-aggressive) and their consequences; discussion of situations where assertive } \\
\text { communication is particularly needed in the perinatal period and within the COVID-19 pandemic; } \\
\text { strategies for increasing assertive communication (e.g., planning for a strategic approach; assertiveness } \\
\text { script; broken record technique). Wrap-up and summary of learning; strategies for relapse prevention. }\end{array}$ \\
\hline
\end{tabular}

NOTE: A detailed description of session-by-session content of the CBGT protocol can be found in our published manual [33]. CBT: cognitive behavioral therapy; CBGT: cognitive behavioral group therapy.

\subsubsection{Edinburgh Postnatal Depression Scale (EPDS)}

The EPDS [48] is a 10-item self-report questionnaire that assesses depression in both pregnant and postpartum women [49]. Items are scored on a 4-point Likert scale, ranging from 0 to 3, with higher scores indicating greater severity of depressive symptoms. The EPDS has demonstrated good sensitivity and specificity for a diagnosis of Major Depressive Disorder. Clinical cut-off scores of 15 or higher during pregnancy, and 13 or higher during the postpartum, are indicative of Major Depressive Disorder [50]. The internal consistency of the EPDS in this sample was $\alpha=0.79$.

\subsubsection{The Client Satisfaction Questionnaire (CSQ)}

The CSQ [51] is a general self-report measure of satisfaction with health and human services, which can be used for a variety of settings. It elicits the client's perspective on the value of services received. It consists of eight items that are to be answered on a 4-point Likert scale, and the overall score consists of item responses summed with a range from 8 to 32. Higher scores indicate higher satisfaction. This scale demonstrates good psychometric properties with high internal consistency (Cronbach's alpha ranging from 0.92 to 0.93 ). In 
terms of construct validity, tests of global improvement correlated with client satisfaction $(r=0.53$ [52]). The internal consistency of the CSQ in this sample was $\alpha=0.89$.

\subsection{Statistical Analysis}

Comparison of demographic and psychological variables, GAD-7, PSWQ, IUS and EPDS scores, between pregnant and postpartum participants, were assessed using independent $t$-tests (for continuous variables) and chi-square tests (for categorical variables). If continuous variables did not meet the assumptions of normality, as assessed by the ShapiroWilk test, the non-parametric Mann-Whitney U test was used. Repeated measures analysis of covariance assessed pre- to post-treatment and follow-up effects on primary (GAD-7) and secondary (PSWQ, IUS, EPDS) outcomes. No mathematical corrections were made for multiple comparisons. Effect sizes for all statistical tests were calculated. For $t$-tests, Cohen's d values of $0.2,0.5$, and 0.8 are considered small, medium, and large, respectively. For chi-square tests and $z$-tests, phi and $\mathrm{r}$ values of $\pm 0.1,0.3$, and 0.5 , are considered small, medium, and large, respectively. All analyses were performed using IBM SPSS Statistics 23 statistical software [53].

\section{Results}

\subsection{Participant Characteristics at Baseline}

Participant demographics and characteristics are reported in Table 1. Pregnant participants ranged between 14 and 40 gestational weeks, with a mean of $30.06(\mathrm{SD}=6.95)$ weeks. Postpartum participants ranged from 1-52 weeks postpartum, with a mean of 20.06 $(\mathrm{SD}=11.82)$ weeks. There were no significant differences $(p>0.05)$ between pregnant and postpartum women with regard to age, ethnicity, marital status, education, comorbid mood or anxiety disorders, or psychotropic medication use. Understandably, postpartum women had significantly more children than pregnant women $(p<0.01)$.

There were no statistically significant or clinically meaningful differences in GAD (GAD-7), worry (PSWQ), or depression (EPDS) symptomatology between pregnant and postpartum women at baseline $(p>0.05)$, with both groups scoring above the clinical threshold on each of these measures. Notably, intolerance of uncertainty (IUS) was significantly higher in pregnant women $\left(\mathrm{M}_{\text {pre }}=92.39\right)$ compared to postpartum women $\left(\mathrm{M}_{\text {pre }}=79.91\right.$; $\mathrm{t}=2.59, p=0.01, \mathrm{~d}=0.70)$. Given this distinction, pre- to post-treatment and follow-up analyses were conducted separately for pregnant and postpartum women.

\subsection{Treatment Engagement and Discontinuation}

In the present study, 68 ( $n=19$ pregnant and $n=49$ postpartum) participants provided informed consent to participate. Fourteen participants $(n=4$ pregnant and $n=10$ postpartum) did not complete the augmented CBGT. Drop-outs were defined as those who completed less than seven treatment sessions or did not provide post-treatment data. Reasons for participant drop-outs were as follows: (1) loss of contact ( $n=3$ pregnant and $n=4$ postpartum); (2) unable to make time commitment ( $n=3$ postpartum); (3) preferred individual services ( $n=2$ postpartum); (4) medical problem requiring attention ( $n=1$ pregnant); and (5) symptoms improved ( $n=1$ postpartum). The difference in participant drop-outs between pregnant and postpartum groups was not statistically significant $\left[\chi^{2}(1)=5.60\right.$, $p=0.23$ ]. Of the 14 pregnant participants who completed group treatment, 10 transitioned to postpartum by the post-treatment assessment, and an additional 3 transitioned by the 3-month follow-up.

\subsection{Pre to Post-Treatment}

\subsubsection{Primary Outcome}

All pre- to post-treatment outcomes for pregnant and postpartum women are presented in Table 3. Both pregnant and postpartum women demonstrated statistically significant and clinically meaningful reductions in GAD symptoms from pre- to post-treatment. 
There was no significant medication use by GAD symptom interactions from pre- to posttreatment in pregnant or postpartum women (all $p$-values $>0.05$ ).

Table 3. Pre- to Post-Treatment Symptom Change in Pregnant $(n=15)$ and Postpartum $(n=39)$ Participants.

\begin{tabular}{|c|c|c|c|c|c|c|c|c|}
\hline & \multicolumn{2}{|c|}{ Pre-Treatment } & \multicolumn{2}{|c|}{ Post-Treatment } & \multirow[b]{2}{*}{ F-Value } & \multirow[b]{2}{*}{$p$-Value } & \multirow[b]{2}{*}{$\eta^{2} p$} & \multirow[b]{2}{*}{$\begin{array}{l}\text { Clinical } \\
\text { Cut-Offs }\end{array}$} \\
\hline & Mean & SD & Mean & SD & & & & \\
\hline \multicolumn{9}{|c|}{ Pregnant } \\
\hline GAD-7 ${ }^{a}$ & 11.64 & 4.89 & 8.93 & 4.29 & 5.01 & 0.045 & 0.295 & 10 \\
\hline PSWQ & 68.87 & 7.75 & 62.20 & 9.53 & 9.79 & 0.08 & 0.430 & 65 \\
\hline EPDS & 15.20 & 4.46 & 10.40 & 4.67 & 17.94 & 0.001 & 0.580 & 15 \\
\hline IUS $^{\mathrm{a}}$ & 93.50 & 20.76 & 83.50 & 19.14 & 9.49 & 0.010 & 0.442 & 64 \\
\hline \multicolumn{9}{|c|}{ Postpartum } \\
\hline GAD-7 b & 12.53 & 4.90 & 6.39 & 3.19 & 44.89 & 0.000 & 0.569 & 10 \\
\hline PSWQ & 66.51 & 8.09 & 59.74 & 9.57 & 22.31 & 0.000 & 0.376 & 65 \\
\hline EPDS & 14.03 & 3.63 & 10.13 & 3.50 & 23.56 & 0.000 & 0.389 & 13 \\
\hline IUS $^{c}$ & 82.14 & 15.68 & 72.41 & 14.45 & 15.50 & 0.000 & 0.307 & - \\
\hline
\end{tabular}

a 1 pregnant participant did not complete the GAD-7 and IUS at post-treatment; ${ }^{\mathrm{b}} 3$ postpartum participants did not complete the GAD-7 at post-treatment; ${ }^{\mathrm{c}} 2$ postpartum participants did not complete the IUS at post-treatment.

\subsubsection{Secondary Outcomes}

With regard to mean clinical change in secondary symptom outcomes, there were no statically significant reductions in worry symptoms for pregnant women from pre- to post-treatment $(p=0.08)$. Pregnant women however, demonstrated clinically meaningful reductions in worry symptoms, as post-treatment PSWQ scores were below the clinical threshold for this measure. Pregnant women showed statistically significant and clinically meaningful reductions in depression $(p=0.001)$, and statistically significant reductions in intolerance of uncertainty symptoms $(p=0.010)$ from pre- to post-treatment. Postpartum women demonstrated statistically significant and clinically meaningful reductions in worry $(p<0.001)$ and depression $(p<0.001)$, as well as statistically significant reductions in intolerance of uncertainty symptoms $(p<0.001)$ from pre- to post-treatment. There was no significant medication use by worry, depression, or intolerance of uncertainty symptom interactions from pre- to post-treatment in pregnant or postpartum women $(p>0.05)$.

\subsection{Post-Treatment to 3-Months Follow-Up}

All symptom improvements made by pregnant and postpartum women during treatment were maintained from post-treatment to 3-months follow-up (see Table 4). Specifically, there were no significant differences in GAD symptoms (GAD-7: $p=1.00$ ), worry (PSWQ: $p=0.13$ ), depression (EPDS: $p=0.27$ ), or intolerance of uncertainty (IUS: $p=0.31$ ) for pregnant women, suggesting that treatment gains were maintained at 3-months follow-up. Similarly, there were no significant differences in GAD symptoms (GAD-7: $p=0.52$ ), worry (PSWQ: $p=0.14$ ), depression (EPDS: $p=0.43$ ), or intolerance of uncertainty (IUS: $p=0.54$ ) for postpartum women from post-treatment to 3-months follow-up. There were no significant medication use by GAD symptom, worry, depression, or intolerance of uncertainty interactions from post-treatment to 3-months follow-up in pregnant or postpartum women (all $p$-values $>0.05)$. 
Table 4. Post-Treatment to Follow-up Symptom Change in Pregnant $(n=11)$ and Postpartum $(n=24)$ Participants.

\begin{tabular}{|c|c|c|c|c|c|c|c|c|}
\hline & \multicolumn{2}{|c|}{ Post-Treatment } & \multicolumn{2}{|c|}{ 3-Month Follow-Up } & \multirow[b]{2}{*}{ F-Value } & \multirow[b]{2}{*}{$p$-Value } & \multirow[b]{2}{*}{$\eta^{2} p$} & \multirow[b]{2}{*}{$\begin{array}{l}\text { Clinical } \\
\text { Cut-Offs }\end{array}$} \\
\hline & Mean & SD & Mean & SD & & & & \\
\hline \multicolumn{9}{|c|}{ Pregnant } \\
\hline GAD-7 ${ }^{a}$ & 7.56 & 3.21 & 7.22 & 2.73 & 0.000 & 1.000 & 0.000 & 10 \\
\hline PSWQ & 59.09 & 8.81 & 59.27 & 8.67 & 2.74 & 0.133 & 0.233 & 65 \\
\hline EPDS & 9.55 & 4.16 & 8.64 & 3.75 & 1.34 & 0.276 & 0.130 & 15 \\
\hline IUS $^{\text {a }}$ & 83.18 & 20.34 & 81.09 & 20.37 & 1.15 & 0.312 & 0.113 & - \\
\hline \multicolumn{9}{|c|}{ Postpartum } \\
\hline GAD-7 ${ }^{b}$ & 6.29 & 2.35 & 7.43 & 3.47 & 0.42 & 0.526 & 0.022 & 10 \\
\hline PSWQ $^{\mathrm{c}}$ & 61.70 & 9.36 & 61.00 & 8.45 & 2.30 & 0.145 & 0.009 & 65 \\
\hline EPDS & 9.70 & 2.57 & 10.75 & 4.29 & 0.64 & 0.432 & 0.028 & 13 \\
\hline IUS $^{\mathrm{c}}$ & 75.33 & 14.86 & 76.86 & 19.29 & 0.38 & 0.543 & 0.020 & - \\
\hline
\end{tabular}

a 2 pregnant participants did not complete the GAD-7 and IUS at 3-month follow-up, ${ }^{\mathrm{b}} 3$ postpartum participants did not complete the GAD-7 at 3-month follow-up; ${ }^{\mathrm{c}} 1$ postpartum participant did not complete the PSWQ and IUS at 3-month follow-up.

\subsection{Treatment Satisfaction and Qualitative Outcomes}

Treatment satisfaction was assessed at post-treatment and is reported for 14 pregnant women and 37 postpartum women. The majority of participants rated the treatment as 'excellent' ( $n=7$ pregnant, $50 \% ; n=23$ postpartum, $62.2 \%$ ) or 'good' ( $n=7$ pregnant, $50 \%$; $n=13$ postpartum $35.1 \%$ ), reported that the treatment 'helped' ( $n=8$ pregnant, $57.1 \%$; $n=6$ postpartum, $16.2 \%$ ) or 'helped a great deal' ( $n=6$ pregnant, $42.9 \% ; n=12$ postpartum, $32.4 \%)$, and reported that they would recommend the treatment to others. The majority of pregnant $(n=13,92.9 \%)$ and postpartum $(n=35,94.6 \%)$ women reported being satisfied with the program.

Qualitative data collected at post-treatment also suggested that $71.7 \%$ of participants ( $n=9$ pregnant, $69.2 \% ; n=24$ postpartum, $72.7 \%$ ) were no longer experiencing excessive anxiety related to COVID-19 by the end of treatment. Similarly, $93.2 \%$ of participants ( $n=12$ pregnant, $100 \% ; n=29$ postpartum, $90.6 \%$ ) reported that the treatment addressed their worry content.

\section{Discussion}

Rates of generalized anxiety symptoms in perinatal women have increased substantially since the onset of COVID [54,55], with evidence indicating that the pandemic has uniquely affected the worry content and lives of perinatal women $[4,5,19,20]$, resulting in reduced medical and social supports, as well as increased uncertainty. Given that no evidence-based psychological treatments have been developed that specifically target COVID-related worry and its impact, the objective of our study was to augment our current evidence-based CBGT for perinatal anxiety protocol $[33,34]$ and evaluate its effectiveness in meeting the unique mental health needs of perinatal women with GAD during the pandemic.

There were no significant differences in baseline GAD, worry or depression symptomatology between pregnant and postpartum women, with both groups scoring above the clinical cut-offs on these respective measures. Notably, intolerance of uncertainty was significantly higher in pregnant women compared to postpartum women, warranting the separation of these groups for pre- to post-treatment and follow-up analyses. Given the already existing uncertainty during pregnancy and the associated risk with postpartum anxiety worsening [23], it is understandable as to why intolerance of uncertainty would be heightened in pregnant women versus postpartum women. Factors which may not have been as relevant for postpartum women during the pandemic, such as reductions in 
perinatal medical supports (e.g., decrease in frequency of appointments, partners unable to join medical appointments) may have acted as an additional contributor to increases in uncertainty beyond those seen in postpartum women.

Consistent with our hypotheses, augmented CBGT led to statistically significant and clinically meaningful reductions in GAD symptoms from pre- to post-treatment, with both pregnant and postpartum women scoring below the clinical threshold following treatment. Importantly, reductions in GAD symptoms were maintained at 3-months follow-up and these gains remained significant while controlling for psychotropic medication use. The magnitude of change in GAD symptoms from pre- to -post-treatment is comparable to other studies looking at the effectiveness of cognitive behavioural therapy for GAD in the general population [56-58] and in perinatal samples [20,34,59]. Further, our qualitative data demonstrating that nearly three quarters of participants reported no longer experiencing excessive anxiety related to COVID-19 by the end of treatment and nearly all participants reported that the augmented CBGT addressed their worry content is a particularly important study outcome. As described, worry content in GAD changes with context and circumstance and research has captured this phenomenon with perinatal women during the COVID-19 pandemic. The fact that nearly all participants reported that treatment addressed their worry content and that most participants no longer had excessive worry related to the pandemic, adds further validation to this protocol's effectiveness.

Our augmented CBGT protocol also led to statistically significant and clinically meaningful reductions in secondary outcomes of intolerance of uncertainty and depression from pre- to post-treatment in pregnant women and clinically meaningful but not statistically significant reductions in worry. Postpartum women demonstrated statistically significant and clinically meaningful reductions in all secondary outcomes (i.e., worry, depression, intolerance of uncertainty) from pre- to post-treatment. Again, gains on all secondary outcomes were maintained at 3-months follow-up for pregnant and postpartum women and were not influenced by psychotropic medication use. Given that worry is a defining feature of GAD [11], and has been linked to maintenance of GAD symptoms [20,59,60], reduction of worry symptoms in our sample is promising.

With regard to depression symptomatology, depression is highly comorbid with GAD [61-63]. Further, depression occurring in pregnancy and the postpartum period has been linked to the development of adverse cognitive, social and emotional outcomes in offspring [64]. As such, effectively targeting depression during treatment for GAD is imperative. The fact that our protocol led to statistically and clinically meaningful reductions in depression symptoms for pregnant and postpartum women from pre- to post-treatment that were maintained at 3 months follow-up is encouraging, especially given pandemic-related physical distancing, lockdown, and social isolation restrictions issued by the government throughout this time.

Reductions in, and maintenance of intolerance of uncertainty symptoms in pregnant and postpartum women is another critical finding from our study, as intolerance of uncertainty in pregnancy has been linked to higher risk of anxiety worsening postnatally [23]. Further, emerging research suggests that more people are struggling with uncertainty during the pandemic [27], which has been associated with increased generalized anxiety, depression, and other mental health symptoms, as well as maladaptive coping strategies, both in the general population [27] and perinatally [28]. Although replication of our findings is necessary, our study provides support for the inclusion of strategies that specifically address intolerance of uncertainty in perinatal women, such as behavioural experiments and mindfulness, included in the present study.

The present study has several limitations. First, we did not use a comparison condition and our sample size was relatively small, which limits generalizability and interpretation of results. As such, future studies should consider evaluating augmented CBGT in a larger sample and against a waitlist-control or other active comparison conditions to further establish its effectiveness. In addition, given the small sample size and limited number of pregnant women in our sample by post-treatment and follow-up, we were unable to 
evaluate what effect gestational age or weeks postpartum had on treatment outcomes. Future studies should consider evaluating whether participating in treatment at various times throughout the perinatal period affects treatment effectiveness. Further, since we did not evaluate the effectiveness of our traditional 6-session CBGT protocol with perinatal women with GAD during the pandemic, we are not certain as to whether that protocol would have been less effective, as effective, or even more effective than our augmented CBGT protocol. In addition, although the present study evaluated maintenance of treatment gains over a three-month period, future studies would benefit from assessing the longer-term impact of the augmented CBGT on primary and secondary outcome measures. Given that the sociodemographic characteristics of our sample were relatively homogenous (i.e., Caucasian, married, highly educated), it is plausible that our augmented CBGT may not be effective for perinatal women of diverse sociodemographic backgrounds. Future studies should evaluate the effectiveness of our augmented CBGT in heterogenous samples of perinatal women. Finally, participants were allowed to use psychotropic medications during this study thus making our sample ecologically valid. While we found no medication use by symptom improvement interaction in our analyses, a future medication-free study could also be very informative.

\section{Conclusions}

To our knowledge, this was the first study to examine the effectiveness of an augmented CBGT for perinatal GAD that is tailored to address the unique mental health needs of pregnant and postpartum women during the COVID-19 pandemic. Our study provides empirical support for a brief, eight-session augmented CBGT protocol to address symptoms of GAD, worry, intolerance of uncertainty, and depression in pregnant and postpartum women irrespective of psychotropic medication use during the COVID-19 pandemic.

Author Contributions: All authors contributed to the conceptualization of the study. S.M.G., M.F. and B.I. contributed to the methodology, data collection, and formal analysis. S.M.G., M.F. and B.I. contributed to the original draft preparation and all authors contributed to the review and editing of the manuscript. All authors have read and agreed to the published version of the manuscript.

Funding: This research was funded in part by an unrestricted educational gift from Shoppers Drug Mart (Run for Women).

Institutional Review Board Statement: The study was conducted according to the guidelines of the Declaration of Helsinki and reviewed and approved by the Hamilton Integrated Research Ethics Board (HIREB) study number 11455, approval dated 11 August 2020.

Informed Consent Statement: Informed consent was obtained from all participants involved in the study.

Data Availability Statement: Data made available upon request due to restrictions (e.g., privacy or ethical).

Acknowledgments: The authors would like to sincerely thank Emma Stallwood, Research Assistant within the Women's Health Concerns Clinic, for helping with the data collection and organization of treatment groups over the course of this study and Hannah Armstrong for helping organize the qualitative data associated with this study. Finally, we would like to thank the women who participated in this study for their time and effort.

Conflicts of Interest: The authors declare no conflict of interest. The funders had no role in the design of the study; in the collection, analyses, or interpretation of data; in the writing of the manuscript, or in the decision to publish the results.

\section{References}

1. Parfitt, Y.; Ayers, S. Transition to parenthood and mental health in first-time parents. Infant Ment. Health J. 2014, 35, 263-273. [CrossRef]

2. Redshaw, M.; Henderson, J. Who is actually asked about their mental health in pregnancy and the postnatal period? Findings from a national survey. BMC Psychiatry 2016, 16, 322. [CrossRef] 
3. Fawcett, E.J.; Fairbrother, N.; Cox, M.L.; White, I.; Fawcett, J.M. The Prevalence of Anxiety Disorders during Pregnancy and the Postpartum Period: A Multivariate Bayesian Meta-Analysis. J. Clin. Psychiatry 2019, 80, 18r12527. [CrossRef] [PubMed]

4. Farewell, C.V.; Jewell, J.; Walls, J.; Leiferman, J.A. A Mixed-Methods Pilot Study of Perinatal Risk and Resilience during COVID-19. J. Prim. Care Community Health 2020, 11, 2150132720944074. [CrossRef]

5. Preis, H.; Mahaffey, B.; Heiselman, C.; Lobel, M. Vulnerability and resilience to pandemic-related stress among U.S. women pregnant at the start of the COVID-19 pandemic. Soc. Sci. Med. 2020, 266, 113348. [CrossRef]

6. Pavlov, M.; Steiner, N.; Kessous, R.; Weintraub, A.Y.; Sheiner, E. Obstetric and neonatal outcome in patients with anxiety disorders J. Matern. Neonatal Med. 2013, 27, 1339-1342. [CrossRef]

7. Uguz, F.; Sonmez, E.O.; Sahingoz, M.; Gokmen, Z.; Basaran, M.; Gezginc, K.; Sonmez, G.; Kaya, N.; Erdem, S.S.; Cicekler, H.; et al. Maternal generalized anxiety disorder during pregnancy and fetal brain development: A comparative study on cord blood brain-derived neurotrophic factor levels. J. Psychosom. Res. 2013, 75, 346-350. [CrossRef]

8. Polte, C.; Junge, C.; Von Soest, T.; Seidler, A.; Eberhard-Gran, M.; Garthus-Niegel, S. Impact of Maternal Perinatal Anxiety on Social-Emotional Development of 2-Year-Olds, A Prospective Study of Norwegian Mothers and Their Offspring: The Impact of Perinatal Anxiety on Child Development. Matern. Child Health J. 2019, 23, 386-396. [CrossRef]

9. Field, T. Prenatal anxiety effects: A review. Infant Behav. Dev. 2017, 49, 120-128. [CrossRef]

10. Prenoveau, J.M.; Craske, M.G.; West, V.; Giannakakis, A.; Zioga, M.; Lehtonen, A.; Davies, B.; Netsi, E.; Cardy, J.; Cooper, P.; et al. Maternal postnatal depression and anxiety and their association with child emotional negativity and behavior problems at two years. Dev. Psychol. 2017, 53, 50-62. [CrossRef]

11. American Psychiatry Association. Diagnostic and Statistical Manual of Mental Disorders, 5th ed.; American Psychiatric Association: Arlington, TX, USA, 2013.

12. Dennis, C.-L.; Falah-Hassani, K.; Shiri, R. Prevalence of antenatal and postnatal anxiety: Systematic review and meta-analysis. Br. J. Psychiatry 2017, 210, 315-323. [CrossRef] [PubMed]

13. Di Nardo, P.A.; Brown, T.A.; Barlow, D.H. Anxiety Disorder Interview Schedule for DSM-IV: Lifetime Version (ADIS-IV-L); Oxford University Press: Oxford, UK, 1994.

14. Constans, I.J.; Barbee, J.G.; Townsend, M.H.; Leffler, H. Stability of worry content in GAD patients: A descriptive study. J. Anxiety Disord. 2002, 16, 311-319. [CrossRef]

15. Correa, J.K.; Brown, T.A. Expression of Generalized Anxiety Disorder across the Lifespan. J. Psychopathol. Behav. Assess. 2018, 41, 53-59. [CrossRef]

16. Diefenbach, G.J.; Stanley, M.A.; Beck, J.G. Worry content reported by older adults with and without generalized anxiety disorder. Aging Ment. Health 2001, 5, 269-274. [CrossRef]

17. Goldfinger, C.; Green, S.M.; Furtado, M.; McCabe, R.E. Characterizing the nature of worry in a sample of perinatal women with generalized anxiety disorder. Clin. Psychol. Psychother. 2019, 27, 136-145. [CrossRef] [PubMed]

18. Taylor, S. The Psychology of Pandemics: Preparing for the Next Global Outbreak of Infectious Disease, 1st ed.; Cambridge Scholars Publishing: Newcastle upon Tyne, UK, 2019.

19. Chivers, B.R.; Garad, R.M.; Boyle, J.A.; Skouteris, H.; Teede, H.J.; Harrison, C.L. Perinatal distress during COVID-19: A thematic analysis of an online parenting forum. J. Med. Internet Res. 2020, 22, e22002. [CrossRef] [PubMed]

20. Green, S.M.; Furtado, M.; Inness, B.E.; Frey, B.N.; McCabe, R.E. Characterizing Worry Content and Impact in Pregnant and Postpartum Women with Anxiety Disorders during COVID-19. Clin. Psychol. Psychother. 2021. [CrossRef] [PubMed]

21. Biaggi, A.; Conroy, S.; Pawlby, S.; Pariante, C.M. Identifying the women at risk of antenatal anxiety and depression: A systematic review. J. Affect. Disord. 2015, 191, 62-77. [CrossRef] [PubMed]

22. Ford, E.; Ayers, S. Stressful events and support during birth: The effect on anxiety, mood and perceived control. J. Anxiety Disord. 2009, 23, 260-268. [CrossRef] [PubMed]

23. Furtado, M.; Van Lieshout, R.J.; Van Ameringen, M.; Green, S.M.; Frey, B.N. Biological and psychosocial predictors of anxiety worsening in the postpartum period: A longitudinal study. J. Affect. Disord. 2019, 250, 218-225. [CrossRef]

24. Buhr, K.; Dugas, M.J. The role of fear of anxiety and intolerance of uncertainty in worry: An experimental manipulation. Behav. Res. Ther. 2009, 47, 215-223. [CrossRef] [PubMed]

25. Buhr, K.; Dugas, M.J. Investigating the construct validity of intolerance of uncertainty and its unique relationship with worry. J. Anxiety Disord. 2006, 20, 222-236. [CrossRef] [PubMed]

26. Laugesen, N.; Dugas, M.J.; Bukowski, W.M. Understanding Adolescent Worry: The Application of a Cognitive Model. J. Abnorm. Child Psychol. 2003, 31, 55-64. [CrossRef] [PubMed]

27. Gentes, E.L.; Ruscio, A.M. A meta-analysis of the relation of intolerance of uncertainty to symptoms of generalized anxiety disorder, major depressive disorder, and obsessive-compulsive disorder. Clin. Psychol. Rev. 2011, 31, 923-933. [CrossRef] [PubMed]

28. Rettie, H.; Daniels, J. Coping and tolerance of uncertainty: Predictors and mediators of mental health during the COVID-19 pandemic. Am. Psychol. 2021, 76, 427-437. [CrossRef]

29. Sbrilli, M.D.; Haigler, K.; Laurent, H.K. The Indirect Effect of Parental Intolerance of Uncertainty on Perinatal Mental Health via Mindfulness during COVID-19. Mindfulness 2021, 12, 1999-2008. [CrossRef] [PubMed]

30. Dugas, M.J.; Ladouceur, R. Treatment of GAD: Targeting Intolerance of Uncertainty in Two Types of Worry. Behav. Modif. 2000, 24, 635-657. [CrossRef] 
31. Zemestani, M.; Beheshti, N.; Rezaei, F.; van der Heiden, C.; Kendall, P.C. Cognitive Behavior Therapy Targeting Intolerance of Uncertainty Versus Selective Serotonin Reuptake Inhibitor for Generalized Anxiety Disorder: A Randomized Clinical Trial. Behav. Chang. 2021, 38, 250-262. [CrossRef]

32. Kim, M.K.; Lee, K.S.; Kim, B.; Choi, T.K.; Lee, S.-H. Impact of Mindfulness-Based Cognitive Therapy on Intolerance of Uncertainty in Patients with Panic Disorder. Psychiatry Investig. 2016, 13, 196-202. [CrossRef] [PubMed]

33. Green, S.M.; Frey, B.N.; Donegan, E.; McCabe, R.E. Cognitive Behavioral Therapy for Anxiety and Depression during Pregnancy and Beyond: How to Manage Symptoms and Maximize Well-Being, 1st ed.; Routledge: New York, NY, USA, 2019.

34. Green, S.M.; Donegan, E.; McCabe, R.; Streiner, D.L.; Agako, A.; Frey, B.N. Cognitive behavioral therapy for perinatal anxiety: A randomized controlled trial. Aust. N. Z. J. Psychiatry 2020, 54, 423-432. [CrossRef]

35. Caropreso, L.; Saliba, S.; Hasegawa, L.; Lawrence, J.; Davey, C.J.; Frey, B.N. Quality assurance assessment of a specialized perinatal mental health clinic. BMC Pregnancy Childbirth 2020, 20, 485. [CrossRef] [PubMed]

36. Sheehan, D.V. Mini International Neuropsychiatric Interview (MINI), English Version 7.0.2 for DSM-5. Available online: https:// harmresearch.org/index.php/product/mini-international-neuropsychiatric-interview-mini-7-0-2-4/ (accessed on 19 December 2021)

37. Hebert, E.A.; Dugas, M.J. Behavioral Experiments for Intolerance of Uncertainty: Challenging the Unknown in the Treatment of Generalized Anxiety Disorder. Cogn. Behav. Pract. 2018, 26, 421-436. [CrossRef]

38. Robichaud, M. Cognitive Behavior Therapy Targeting Intolerance of Uncertainty: Application to a Clinical Case of Generalized Anxiety Disorder. Cogn. Behav. Pract. 2012, 20, 251-263. [CrossRef]

39. Spitzer, R.L.; Kroenke, K.; Williams, J.B.W.; Löwe, B. A Brief Measure for Assessing Generalized Anxiety Disorder: The GAD-7. Arch. Intern. Med. 2006, 166, 1092-1097. [CrossRef] [PubMed]

40. Meyer, T.J.; Miller, M.L.; Metzger, R.L.; Borkovec, T.D. Development and validation of the penn state worry questionnaire. Behav. Res. Ther. 1990, 28, 487-495. [CrossRef]

41. Fresco, D.; Mennin, D.S.; Heimberg, R.G.; Turk, C.L. Using the Penn State Worry Questionnaire to identify individuals with generalized anxiety disorder: A receiver operating characteristic analysis. J. Behav. Ther. Exp. Psychiatry 2003, 34, 283-291. [CrossRef] [PubMed]

42. Brown, T.A.; Antony, M.M.; Barlow, D.H. Psychometric properties of the Penn state worry questionnaire in a clinical anxiety disorders sample. Behav. Res. Ther. 1992, 30,33-37. [CrossRef]

43. Blackmore, E.R.; Gustafsson, H.; Gilchrist, M.; Wyman, C.; O'Connor, T.G. Pregnancy-related anxiety: Evidence of distinct clinical significance from a prospective longitudinal study. J. Affect. Disord. 2016, 197, 251-258. [CrossRef] [PubMed]

44. Swanson, L.M.; Pickett, S.; Flynn, H.; Armitage, R. Relationships among Depression, Anxiety, and Insomnia Symptoms in Perinatal Women Seeking Mental Health Treatment. J. Women's Health 2011, 20, 553-558. [CrossRef] [PubMed]

45. Buhr, K.; Dugas, M. The intolerance of uncertainty scale: Psychometric properties of the English version. Behav. Res. Ther. 2002, 40, 931-945. [CrossRef]

46. Freeston, M.H.; Rhéaume, J.; Letarte, H.; Dugas, M.; Ladouceur, R. Why do people worry? Pers. Individ. Differ. 1994, 17, 791-802. [CrossRef]

47. Furtado, M.; Frey, B.N.; Green, S.M. Validation of the intolerance of uncertainty scale as a screening tool for perinatal anxiety. BMC Pregnancy Childbirth 2021, 21, 1-10. [CrossRef]

48. Cox, J.L.; Holden, J.M.; Sagovsky, R. Detection of Postnatal Depression. Development of the 10-Item Edinburgh Postnatal Depression Scale. Br. J. Psychiatry 1987, 150, 782-786. [CrossRef] [PubMed]

49. Murray, D.; Cox, J.L. Screening for depression during pregnancy with the edinburgh depression scale (EDDS). J. Reprod. Infant Psychol. 1990, 8, 99-107. [CrossRef]

50. Matthey, S.; Henshaw, C.; Elliott, S.; Barnett, B. Variability in use of cut-off scores and formats on the Edinburgh Postnatal Depression Scale-implications for clinical and research practice. Arch. Women's Ment. Health 2006, 9, 309-315. [CrossRef]

51. Larsen, D.L.; Attkisson, C.; Hargreaves, W.A.; Nguyen, T.D. Assessment of client/patient satisfaction: Development of a general scale. Eval. Program Plan. 1979, 2, 197-207. [CrossRef]

52. LeVois, M.; Nguyen, T.D.; Attkisson, C. Artifact in client satisfaction assessment: Experience in community mental health settings. Eval. Program Plan. 1981, 4, 139-150. [CrossRef]

53. IBM Corp. IBM SPSS Statistics for Windows, Version 23; IBM Corp.: Armonk, NY, USA, 2015.

54. Cameron, E.E.; Joyce, K.; Delaquis, C.; Reynolds, K.; Protudjer, J.L.; Roos, L.E. Maternal psychological distress \& mental health service use during the COVID-19 pandemic. J. Affect. Disord. 2020, 276, 765-774. [CrossRef] [PubMed]

55. Liu, D.; Li, L.; Wu, X.; Zheng, D.; Wang, J.; Yang, L.; Zheng, C. Pregnancy and Perinatal Outcomes of Women With Coronavirus Disease (COVID-19) Pneumonia: A Preliminary Analysis. Am. J. Roentgenol. 2020, 215, 127-132. [CrossRef]

56. Cuijpers, P.; Sijbrandij, M.; Koole, S.; Huibers, M.; Berking, M.; Andersson, G. Psychological treatment of generalized anxiety disorder: A meta-analysis. Clin. Psychol. Rev. 2014, 34, 130-140. [CrossRef]

57. Carl, E.; Witcraft, S.M.; Kauffman, B.Y.; Gillespie, E.M.; Becker, E.S.; Cuijpers, P.; Van Ameringen, M.; Smits, J.A.J.; Powers, M.B Psychological and pharmacological treatments for generalized anxiety disorder (GAD): A meta-analysis of randomized controlled trials. Cogn. Behav. Ther. 2019, 49, 1-21. [CrossRef] [PubMed]

58. Loughnan, S.A.; Sie, A.; Hobbs, M.J.; Joubert, A.E.; Smith, J.; Haskelberg, H.; Mahoney, A.E.; Kladnitski, N.; Holt, C.J.; Milgrom, J.; et al. A randomized controlled trial of 'MUMentum Pregnancy': Internet-delivered cognitive behavioral therapy program for antenatal anxiety and depression. J. Affect. Disord. 2018, 243, 381-390. [CrossRef] [PubMed] 
59. Donegan, E.; Dugas, M.J. Generalized anxiety disorder: A comparison of symptom change in adults receiving cognitive-behavioral therapy or applied relaxation. J. Consult. Clin. Psychol. 2012, 80, 490-496. [CrossRef] [PubMed]

60. Mahoney, A.E.; Hobbs, M.J.; Williams, A.D.; Andrews, G.; Newby, J.M. The Mediating Relationship between Maladaptive Behaviours, Cognitive Factors, and Generalised Anxiety Disorder Symptoms. Behav. Chang. 2018, 35, 123-138. [CrossRef]

61. Price, M.; Legrand, A.C.; Brier, Z.M.; Hébert-Dufresne, L. The symptoms at the center: Examining the comorbidity of posttraumatic stress disorder, generalized anxiety disorder, and depression with network analysis. J. Psychiatr. Res. 2018, 109, 52-58. [CrossRef] [PubMed]

62. Moffitt, T.E.; Caspi, A.; Harrington, H.; Milne, B.J.; Melchior, M.; Goldberg, D.; Poulton, R. Generalized anxiety disorder and depression: Childhood risk factors in a birth cohort followed to age 32. Psychol. Med. 2007, 37, 441-452. [CrossRef] [PubMed]

63. Simon, N.M. Generalized anxiety disorder and psychiatric comorbidities such as depression, bipolar disorder, and substance abuse. J. Clin. Psychiatry 2009, 70, 10-14. [CrossRef]

64. Rogers, A.; Obst, S.; Teague, S.J.; Rossen, L.; Spry, E.A.; Macdonald, J.A.; Sunderland, M.; Olsson, C.A.; Youssef, G.; Hutchinson, D. Association Between Maternal Perinatal Depression and Anxiety and Child and Adolescent Development: A Meta-analysis. JAMA Pediatr. 2020, 174, 1082-1092. [CrossRef] 